\title{
Hierarchy and Wave Functions in a Simple Quantum Cosmology
}

\author{
T. R. Mongan * \\ 84 Marin Avenue \\ Sausalito, CA 94965, U.S.A.
}

\begin{abstract}
Astrophysical observations indicate the universe is asymptotic to a de Sitter space with vacuum energy density $\Omega_{A}=0.7$. Then, since $H_{0} \approx 65 \mathrm{~km} \mathrm{sec}^{-1} \mathrm{Mpc}^{-1}$, our universe has only about $10^{122}$ degrees of freedom. A finite number of degrees of freedom is consistent with a closed universe arising from a quantum fluctuation, with zero total quantum numbers. If space-time has eleven dimensions, and the universe began as a closed force-symmetric ten-dimensional space with characteristic length $l$, seven of the space dimensions must have collapsed to generate the three large space dimensions we perceive. The holographic entropy conjecture then suggests the initial length scale $l$ must be roughly twenty orders of magnitude larger than the Planck length. Accordingly, the nuclear force must be about forty orders of magnitude stronger than gravity, possibly resolving the force heirarchy problem. A wavefunction for the radius of curvature of the universe can be obtained from the Schrödinger equation derived by Elbaz and Novello. The product of this wavefunction and its complex conjugate can be interpreted as the probability density for finding a given radius of curvature in one of the infinity of measurements of the radius of curvature possible (in principle) at any location in a homogeneous isotropic universe.
\end{abstract}

*E-mail: tmongan@mail.com 
The nuclear force is $1.7 \times 10^{38}$ times stronger than gravity, and there are indications this force hierarchy relates to the vacuum energy density/cosmological constant. For instance, if the proton mass is specified, the observed vacuum energy density can be calculated from a simple quantum cosmological model [1] of eleven-dimensional space-time. In another approach, Mena Marugan and Carneiro [2] consider a three-dimensional universe dominated by the observed vacuum energy density and apply the holographic conjecture [3] to determine the total number of observable degrees of freedom in the universe. They set this equal to the estimated number of degrees of freedom for elementary excitations of typical size $l_{N}$ inside the maximum observable Hubble radius. They then estimate the proton mass from the length scale $l_{N}$, using the uncertainty principle. The result is about twenty orders of magnitude smaller than the Planck mass, suggesting a nuclear force roughly 40 orders of magnitude stronger than gravity. In contrast, the approach outlined below uses the holographic conjecture to relate the force hierarchy to the existence of seven extra space dimensions.

The Friedmann equation for the radius of curvature $R$ of a closed homogeneous isotropic universe [4, 5] is

$$
\dot{R}^{2}-\left(\frac{8 \pi G}{3}\right)\left[\varepsilon_{r}\left(\frac{R_{0}}{R}\right)^{4}+\varepsilon_{m}\left(\frac{R_{0}}{R}\right)^{3}+\varepsilon_{v}\right]\left(\frac{R}{c}\right)^{2}=-c^{2}
$$

where $\varepsilon_{r}, \varepsilon_{m}, \varepsilon_{v}$ and $R_{0}$ are, respectively, the present values of the radiation, matter and vacuum energy densities and the radius of curvature. Astrophysical measurements indicate the expansion of the universe is accelerating, and the energy density of the universe is dominated by a cosmological constant/vacuum energy density with $\Omega_{\Lambda}=0.7$. The cosmological constant is related to the vacuum energy density [4] by $\Lambda=8 \pi G \varepsilon_{v} / c^{4}$. As $R \rightarrow \infty$, the radiation and matter energy density (and the curvature energy) are driven to zero by the expansion of the universe, and the Friedmann equation becomes

$$
\left(\frac{\dot{R}}{R}\right)^{2}=\frac{8 \pi G \varepsilon_{v}}{c^{2}}=\frac{\Lambda c^{2}}{3} .
$$

So, our universe is asymptotic to a de Sitter space (the vacuum solution to Einstein's equations with a positive cosmological constant). There is a cosmological horizon in a de Sitter space, because no signal can be received from beyond the Hubble radius where the expansion velocity equals the speed of light. The Hubble radius at $\dot{R}=c$, the maximum observable Hubble distance, is $\sqrt{3 / \Lambda}$ and the area of the cosmological horizon is $A=12 \pi / \Lambda$. Then, according to the holographic conjecture, the number of observable degrees of freedom in the universe is $N=A / 4=3 \pi / \Lambda$. For a Hubble constant $H_{0}=65 \mathrm{~km} \mathrm{sec}^{-1} \mathrm{Mpc}^{-1}[\underline{6}$, the critical density $\rho_{c}=3 H_{0}^{2} / 8 \pi G=7.9 \times 10^{-30} \mathrm{~g} \mathrm{~cm}^{-3}$, the vacuum energy density $\varepsilon_{v}=0.7 \rho_{c} c^{2}=5.0 \times 10^{-9} \mathrm{~g} \mathrm{~cm}^{2} \mathrm{sec}^{-2} \mathrm{~cm}^{-3}$, and $\Lambda=1.0 \times 10^{-56} \mathrm{~cm}^{-2}$. So, $N=$ $3 \pi /\left(\Lambda \delta^{2}\right)=3.5 \times 10^{122}$, where $\delta$ is the Planck length. 
A finite number of degrees of freedom is consistent with a closed universe arising from a quantum fluctuation, with zero total quantum numbers. If space-time has eleven dimensions, the simple quantum cosmology in [1] envisions our universe as a closed tendimensional space that is the direct product of a closed three-dimensional subspace and a closed seven-dimensional subspace as the processes that break force symmetry begin. Initially, both spaces have length scale $l$ and radius $l / 2 \pi$, and all forces have equal strength. The length scale is related to the initial strength $G_{i}$ of the nuclear and gravitational force by $l=\sqrt{\hbar G_{i} / c^{3}}$.

In the model in [1], the seven-dimensional subspace collapsed and injected entropy into the three-dimensional subspace, generating the three large space dimensions we inhabit. This suggests that the initial size of the direct product space must be large enough to contain the number of degrees of freedom needed to describe the evolution of our observable universe. According to the holographic conjecture, the number of degrees of freedom available for describing the light sheets of any surface is bounded by one quarter of the area $A$ of the surface in Planck units [7. The characteristic dimension of the initial state of the universe can be estimated by extending the holographic conjecture to physical systems with more than three space dimensions.

In the initial state, the seven-dimensional subspace dominates the contribution of the three-dimensional subspace to the available degrees of freedom because of its higher dimensionality. The area of a seven-sphere [8] of radius $l / 2 \pi$ is $\frac{16 \pi^{3}}{15}\left(\frac{l}{2 \pi \delta}\right)^{6}$ in units of the Planck length $\delta$. Denote the initial surface area of the seven-dimensional subspace by $A_{7}=\frac{16 f \pi^{3}}{15}\left(\frac{l}{2 \pi \delta}\right)^{6}$, where $f$ is greater than one, to allow for the fact that the topology of the seven-dimensional subspace is likely to be considerably more intricate than that of a seven-sphere. If the holographic bound is saturated, so $N=A_{7} / 4$, the number of degrees of freedom in the seven-dimensional subspace is $N=\frac{4 f \pi^{3}}{15}\left(\frac{l}{2 \pi \delta}\right)^{6}$. For $N=3.5 \times 10^{122}, l=\left(1.2 \times 10^{21}\right) f^{-1 / 6} \delta$. This indicates the nuclear force would have to be about forty-two orders of magnitude stronger than gravity to insure that an initial state involving a spherical seven-dimensional subspace $(f=1)$ could contain all the degrees of freedom necessary to describe our observable universe. However, candidate extra dimensional spaces in theories of the fundamental forces governing the universe are far more complex than a sphere (see, e.g., the representations of Calabi-Yau manifolds in [9]). Specification of the detailed nature of the seven extra space dimensions of eleven-dimensional space-time requires a detailed theory of the fundamental forces (e.g., a finite dimensional theory asymptotic to M-theory as the number of degrees of freedom goes to infinity [8]). If, for example, the perimeter of any planar section of the seven-dimensional subspace with characteristic radius $l / 2 \pi$ is $90 l$, the surface area of the subspace will be $5 \times 10^{11}$ times the area of a seven-sphere with radius $l / 2 \pi$. In this case, in eleven-dimensional space-time, an 
initial length scale $l=2.1 \times 10^{-14} \mathrm{~cm}$ (and a nuclear force $1.7 \times 10^{38}$ times stronger than gravity) is necessary to produce an initial seven-dimensional subspace containing enough degrees of freedom to describe our vacuum energy dominated universe with $\Omega_{\Lambda}=0.7$.

On another subject, interpreting the wavefunction of the universe can be problematic in quantum cosmology. In the quantum cosmology outlined in [1], the stationary state wavefunction for the radius of curvature of our three-dimensional universe is obtained from the Schrödinger equation

$$
-\frac{\hbar^{2}}{2 m} \frac{d^{2}}{d R^{2}} \psi-\frac{4 \pi m G}{3}\left(\varepsilon_{m}+\varepsilon_{r}+\varepsilon_{v}\right)\left(\frac{R}{c}\right)^{2} \psi=-E \psi
$$

derived by Elbaz et al [10] and Novello et al [11. The time-dependent wavefunction of the universe is a superposition of stationary state solutions with energies close to the Einstein energy $E=-\frac{1}{2} m c^{2}$. In principle, the radius of curvature of the universe can be measured in an infinity of directions at any point in a homogeneous and isotropic universe. The product of the time-dependent wavefunction and its complex conjugate can be interpreted as the probability density for finding a given value of the radius of curvature in one of these measurements. 


\section{References}

[1] T.R. Mongan, General Relativity and Gravitation 33 (2001) 1415, (gr-qc/0103021).

[2] G. A. Mena Marugan and S. Carneiro, Holography and the Large Number Hypothesis, (gr-qc/0111034).

[3] R. Bousso, JHEP 9907 (1999) 004, (hep-th/9905177).

[4] J. N. Islam, An introduction to mathematical cosmology, Cambridge University Press, 1992.

[5] C. W. Misner, K.S. Thorne, and J. A. Wheeler, Gravitation, W. H. Freeman and Company, New York, 1973.

[6] J. Primack, Cosmological Parameters 2000, talk presented at the 4th international Symposium on Sources and Detection of Dark Matter in the Universe, Marina del Rey, California, 20-23 Feb. 2000, (astro-ph/0007187).

[7] R. Bousso, hep-th/00203101, to be published in Rev. Mod. Phys.

[8] R. Bousso, JHEP 0104 (2001) 035, (hep-th/0012052).

[9] Greene, B., The Elegant Universe, W.W. Norton \& Co., New York, 1999.

[10] E. Elbaz, M. Novello, J. M. Salim, M. C. Motta da Silva and R. Klippert, General Relativity and Gravitation 29 (1997) 481.

[11] M. Novello, J.M. Salim, M.C. Motta da Silva and R. Klippert, Phys. Rev. D54 (1996) 6202. 